\title{
Rumo à visibilidade total da rede: uma estratégia adaptativa para coordenadar a coleta de dados de telemetria in-band
}

\author{
Rumenigue Hohemberger, Fabio Diniz Rossi, Marcelo Caggiani Luizelli \\ ${ }^{1}$ Universidade Federal do Pampa (UNIPAMPA)
}

As redes autônomas (ou self-driving networks) são redes capazes de prever mudanças e se adaptar sem a intervenção humana. Um passo importante em direção a esse paradigma de rede emergente envolve o fechamento do loop de gerenciamento, o qual inclui medir, analisar e controlar a rede continuamente [Kalmbach et al. 2018]. O aumento da automação tem o potencial não apenas de simplificar as operações de rede, mas também de possibilitar otimizações. Uma forma de otimizar o desempenho da rede seria através do roteamento automático do tráfego pelo caminho de melhor desempenho, atendendo as Services Level Agreement - SLAs. Ainda, heavy hitters poderiam ser identificados e bloqueados automaticamente. As redes autônomas têm o potencial de reduzir os custos operacionais e, mais importante, aumentar a flexibilidade da operação das infraestruturas de rede.

Avanços recentes em Aprendizado de Máquina (ML - Machine Learning) abriram o caminho para a adoção de redes autônomas, já que os modelos de ML podem identificar padrões analisando informações e aprender como reagir a mudanças na rede (isto é, controlar) [Boutaba et al. 2018]. No entanto, pouco ainda foi feito para medir e coletar dados em tempo real para subsidiar tais modelos - isto é, a telemetria de rede em tempo real. A telemetria de rede in-band (INT - In-band Network Telemetry) surgiu recentemente como uma alternativa promissora de monitoramento de rede em tempo real para fornecer maior visibilidade aos operadores [Liu et al. 2018]. INT permite coletar e encapsular informações de telemetria de baixo nível no tráfego da rede de produção sempre que possível. Esses dados incluem estados internos do plano de dados( ocupação de fila de entrada do roteador entre outros) e métricas de desempenho de rede ( tempo e latência de processamento do plano de dados), por exemplo. No processo de telemetria in-band, as informações coletadas são transportadas em um pacote ao longo de seu caminho de roteamento e, em algum ponto da rede, são extraídas e relatadas para um aplicativo de monitoramento.

Em contraste com as ferramentas tradicionais de monitoramento e de troubleshooting, INT pode fornecer a visibilidade de rede necessária para identificar (i) comportamentos de rede de curta duração (no caso micro-burst) e (ii) anomalias de rede (por exemplo, violações de roteamento). A rápida identificação de anomalias de rede é primordial para o sucesso da operação de redes autônomas e para a correta operação de aplicações com requisitos estritos de latência como, por exemplo, aplicações em realidade aumentada. $\mathrm{O}$ conceito de telemetria in-band tem se mostrado viável na prática, sendo demonstrados por [Liu et al. 2018, Gupta et al. 2018]) e tem sido amplamente discutida por IETF. É importante mencionar que o surgimento da telemetria in-band foi fomentado por planos de dados programáveis (por exemplo, SmartNICs) e para linguagens de domínio específico como, por exemplo, a linguagem P4 [Bosshart et al. 2014]) .

Esforços tem sido empregados para oferecer o melhor da telemetria inband para melhorar a visibilidade da infraestrutura. Liu et al. [Liu et al. 2018] e Pan et al. [Pan et al. 2019] se concentraram na realização de telemetria de rede através de pacotes INT ativos. Ambas as estratégias utilizam circuitos eulerianos para instruir o caminho dos pacotes enviados. Mais recentemente, Marques et al. [Marques et al. 2019] forneceu abordagens heurísticas para orquestrar como pacotes de produção coletariam dados de telemetria da rede. Embora esses estudos sejam os primeiros passos para otimizar o uso da telemetria in-band, eles não conseguem oferecer uma visibilidade eficaz em toda a rede. A abordagem proposta por Marques et al. [Marques et al. 2019] incorpora 
dados de telemetria no tráfego da rede de produção. Os dados de telemetria podem ser oportunisticamente incorporados em campos de cabeçalho não utilizados de protocolos convencionais (por exemplo, TCP) e, portanto, podem ser transportados ao longo da rota tomada pelo tráfego de rede de produção (e capturar eventos transitórios). Apesar do progresso feito, as heurísticas projetadas são agnósticas para a frequência e consistência dos dados de telemetria coletados, levando (potencialmente) a uma visão imprecisa da rede. As heurísticas assumem que os fluxos de rede são igualmente equilibrados entre os pares de destinos de origem e que a topologia de rede não afeta o processo de telemetria. Portanto, os itens de telemetria em dispositivos de rede específicos (por exemplo, hubs de rede) teriam mais chances de serem coletados com mais frequência do que outros itens de telemetria.

Apesar desses esforços para orquestrar a telemetria de rede em banda em redes programáveis, pouco ainda foi feito para coordenar dinamicamente como coletar informações de rede neste novo paradigma. Diante do exposto, o problema fundamental está em como fornecer uma visibilidade de rede efetiva que poderia ser usada adequadamente como entrada para algoritmos de ML. Neste trabalho, propõem-se uma estratégia adaptativa para a seleção de caminho de roteamento ciente das necessidades de telemetria in-band para ser empregado em infraestruturas de redes programáveis de larga escala. A ideia consiste em rotear os fluxos de rede de maneira adaptativa frente as condições da rede, interferindo minimamente em métricas de desempenho (por exemplo, latência fima-fim). Para isso, neste trabalho apresenta-se o estudo inicial sobre telemetria in-band e os algoritmos e modelos que podem ser adotados para projetar o mecanismo de roteamento necessário.

\section{Referências}

Bosshart, P., Daly, D., Gibb, G., Izzard, M., McKeown, N., Rexford, J., Schlesinger, C., Talayco, D., Vahdat, A., Varghese, G., and Walker, D. (2014). P4: Programming protocol-independent packet processors. ACM SIGCOMM 14, 44(3):87-95.

Boutaba, R., Salahuddin, M. A., Limam, N., Ayoubi, S., Shahriar, N., Estrada-Solano, F., and Caicedo, O. M. (2018). A comprehensive survey on machine learning for networking: evolution, applications and research opportunities. Journal of Internet Services and Applications, 9(1):16.

Gupta, A., Harrison, R., Canini, M., Feamster, N., Rexford, J., and Willinger, W. (2018). Sonata: Query-driven streaming network telemetry. In Proceedings of the 2018 Conference of the ACM Special Interest Group on Data Communication, SIGCOMM '18, pages 357-371, New York, NY, USA. ACM.

Kalmbach, P., Zerwas, J., Babarczi, P., Blenk, A., Kellerer, W., and Schmid, S. (2018). Empowering self-driving networks. In Proceedings of the Afternoon Workshop on SelfDriving Networks, SelfDN 2018, pages 8-14, New York, NY, USA. ACM.

Liu, Z., Bi, J., Zhou, Y., Wang, Y., and Lin, Y. (2018). Netvision: Towards network telemetry as a service. In 2018 IEEE 26th International Conference on Network Protocols (ICNP), pages 247-248.

Marques, J. A., Luizelli, M. C., Da Costa, R. I. T., and Gaspary, L. P. (2019). An optimization-based approach for efficient network monitoring using in-band network telemetry. Journal of Internet Services and Applications, (1):16.

Pan, T., Song, E., Bian, Z., Lin, X., Peng, X., Zhang, J., Huang, T., Liu, B., and Liu, Y. (2019). Int-path: Towards optimal path planning for in-band network-wide telemetry. In IEEE INFOCOM 19, pages 1-9. 